\title{
$\operatorname{CoNF}-960477--7$
}

\section{Symposium on the Application of Geophysics to Engineering and Environmental Problems}

Pes

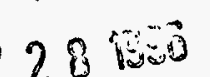

\author{
Compiled by: \\ Ronald S. Bell \\ Mark H. Cramer
}

Min 28 bo

OSTI

April 28 - May 2, 1996

Keystone, Colorado

Sponsored by:

Environmental and Engineering Geophysical Society 


\title{
A new approach to interpretation of airborne magnetic and electromagnetic data.
}

\author{
P. Traynin and M. Zhdanov \\ University of Utah, Department of Geology and Geophysics \\ Salt Lake City, UT 84112 \\ J. Nyquist, L. Beard, W. Doll \\ Environmental Science Division, Oak Ridge National Laboratory, \\ Oak Ridge, TN $37831-6400$
}

\section{Introduction}

We present a new technique for underground imaging based on the idea of space-frequency filtering and downward continuation of the observed airborne magnetic and electromagnetic data. The technique includes two major methods. The first method is related to the downward analytical continuation and is based on the calculation of the total normalized gradient of the observed field. The second method is based on Wiener filtering and takes into account a priori information about typical AEM anomaly shape from a possible target.

\section{Theory of the total normalized gradient}

In recent years a large number of digital AEM data sets representing different geological situations has been obtained. Due to the large amount of data, the interpretation process has to be facilitated by the development of special techniques for fast, semiautomatic analysis of 2-D and 3-D data. In the case of frequency-domain survey interpretation, approaches developed to provide the depth and the geometry of the causative bodies in potential fields based on utilizing the analytical signal or energy envelope, can be used with appropriate modifications. Potential field interpretation methods based on the analytic signal were developed independently by Nabighian $(1972,1974)$ and by Berezkin (1973). Most recently Roest et al. (1992) successfully demonstrated the use of the analytic signal for 3-D aeromagnetic data interpretation.

The main assumption which we make in order to extend the method to time-varing field is a constant separation between a transmitter and a receiver as well as a constant flight altitude. Then the received magnetic field can be approximated as a static potential field, with sources located beneath the Earth's surface (geological inhomogeneities) and above the flight line (the initial field transmitted into the air can be approximated as a plane wave, generated by the infinitely high source). Thus, the observed magnetic field can be considered as a harmonic function. By definition, the analytic signal of any $2-\mathrm{D}$ function $U(x, z)$ is introduced as

$$
A(x, z)=\left[\frac{\partial U(x, z)}{\partial x}\right]+i\left[\frac{\partial U(x, z)}{\partial z}\right] .
$$

The central idea of using the analytic signal in the potential fields interpretation is that it is analytic everywhere except at the sources, where it becomes singular. The downward continuation of the 
analytic signal indicates the source locations and depths. However, the implementation of this idea is greatly complicated by the growth in oscillations of the field with depth, necessitating some form of normalization to regulate these oscillations.

We introduce our normalization of the analytic signal, following Berezkin's method (1973). We call the squared modulus of the analytic signal $A(x, z)$ the total gradient $G(x, z)$. In 2-D case the magnitude of the total gradient of the vertical component of the anomalous field $H_{z a}(x, z)$ is represented by the following equation:

$$
|G(x, z)|=\sqrt{\left[\frac{\partial H_{z a}(x, z)}{\partial x}\right]^{2}+\left[\frac{\partial H_{z a}(x, z)}{\partial z}\right]^{2}} .
$$

Following Berezkin (1973), we will use the normalization of $G(x, z)$ and call it the total normalized gradient $G_{n}(x, z)$ :

$$
G_{n}(x, z)=\frac{|G(x, z)|}{\langle|G(x, z)|>}
$$

where the angle brackets indicate spatial averaging in $\mathrm{x}$.

We use the Poisson equation to compute vertical or horizontal derivatives of the magnetic field $H(x, z)$ at the depth $z$ from the spatial spectrum of the magnetic field at the observation surface $h(w, 0)$ :

$$
H(x, z)=\int_{-\infty}^{+\infty} h(w, 0) e^{-i \omega x} e^{\omega z} d \omega .
$$

where $\omega$ is a spatial frequency.

We can rewrite equation (4) as

$$
H(x, z)=\sum_{k=0}^{\infty} h\left(\omega_{k}\right) e^{-i \omega_{k} x} e^{\omega_{k} z}
$$

where $h\left(\omega_{k}\right)$ are complex Fourier coefficients $h\left(\omega_{k}\right)=A_{k}+i B_{k}$.

In numerical implementation of the algorithm we use the sine Fourier transform, which has better convergence that the cosine transform. However, to use the sine transform, we have to ensure that $H(x, 0)$ is zero at the both ends of the observation interval. To achieve that, we subtract a linear trend from the observed function $H(x, 0)$. We also truncate the infinite Fourier series in equation (5) to the limited number of harmonics $N$, so the sine transform can be expressed as

$$
H(x, z)=\sum_{k=1}^{N} B_{k} \sin ^{\frac{\pi k x}{L}} e^{\frac{\pi k z}{L}},
$$

where $L$ is the observation profile distance, and coefficients $B_{k}$ are expressed using well-known integral formula

$$
B_{k}=\int_{0}^{L} H(x, 0) \sin ^{\frac{\pi k x}{L}} d x .
$$

The accuracy of the procedure depends on two major factors: 
- The length of observation interval $L$.

Numerous synthetic examples indicate, that by satisfying the following condition, errors due to the finite observation profile become negligible:

$$
L \geq 5 h_{\mathrm{d}}
$$

where $h_{d}$ is the possible target's depth.

- The number of Fourier coefficients in the Fourier series.

Reducing the number of harmonics $N$ increases the computation accuracy, but shifts the total normalized gradient maximums downward. We compute the total normalized gradient distribution with several different numbers of Fourier coefficients and then use the empirical rule of thumb that the optimal number of harmonics $N_{\text {opt }}$ is achieved when the maximum $G_{n}\left(N_{\text {opt }}\right)$ is the absolute maximum over all selected number of harmonics.

\section{Application of the total normalized gradient in depth estimations.}

Berezkin (1973), Nabighian (1972) have shown that for potential fields with different sources (singularities) with the coordinates at $\left(x_{s}, z_{s}\right)$ the total normalized gradient $G_{n}(x, z)$ grows as $x$ approaches $x_{s}$ and $z$ approaches $z_{s}$ and becomes singular at the exact location of the source. For depths greater than $z_{s}, G_{n}(x, z)$ decreases, tending to zero at large depths. For isometric objects, singular points coincide with the mass's center, as shown in Figure 1 (left). The very important feature of the $G_{n}(x, z)$ is that it reflects changes in the physical properties of the anomalous bodies. Figure 1 (right) shows the same model as in Figure 1 (left), with the susceptibility of the second body reduced to 125 cgs units from $250 \mathrm{cgs}$ units. As we can see, the value of $G_{n}(x, z)$ maximum over this body also is reduced.

In the electromagnetic case, the standard downward analytic continuation scheme produces a less accurate representation of the field. However even in this case the total normalized gradient is able to produce a reasonable depth estimation for a model which contains a conductive body (resistivity 1 $\mathrm{Ohm}-\mathrm{m})$ in a otherwise resistive $(100 \mathrm{Ohm}-\mathrm{m})$ homogeneous medium. The frequency-domain response ( $4 \mathrm{kHz}$ ) was computed at different flight levels from the earth's surface, up to $50 \mathrm{~m}$ by using the 3-D integral equation code SYSEM (Xiong, 1992). Figures 2 (left) and 2 (right) show the results of using the total normalized gradient method. The depth estimate produced by the method gives a fairly good correspondence with the real depth of the causative body. The depth estimate shifts slightly upward with increasing flight altitude. This is the consequence of using the standard downward continuation technique.

\section{Spatial filtering and downward continuation}

To improve the airborne survey resolution we have used space-frequency spectral analysis of observed geophysical data which allows to interpret a large amount of field data very rapidly. The analysis is a two step process based on a downward continuation transformation. The downward continuation technique is designed to improve the airborne survey resolution since it reduces the distance between anomalous underground structures and an observation point. However from a numerical point of view 
it is an unstable procedure. To reduce the numerical instability Wiener spatial filtering was used. Both steps are carried out in the spatial frequency-domain using a two-dimensional Fourier transform.

\section{Interpretation of airborne geophysical data at Oak Ridge Reservation}

We applied this new interpretational approach to airborne geophysical data collected over the Oak Ridge Reservation. During the last two years the detailed surface and airborne electromagnetic and magnetic surveys have been carried out at the Oak Ridge Reservation (Doll et al., 1995, Beard et al., 1995). The surveys were designed to better characterize large known waste sites and to detect unknown sites. Our modeling study has shown that the EM signature from the prismatic target with the side length less than $10 \mathrm{~m}$, which was used to approximate waste objects, is undetectable by the current airborne EM system (L. Beard et al., 1995). We now apply the two-step procedure described above to reconnaissance airborne magnetic data from the SWSA 6 area. The pole reduced magnetic map of the SWSA 6 area is shown in Figure 3 (left). Spatial filtering was applied to the downward continued field, and was designed to remove magnetic signatures of all targets less than 10 meters in radius. The results are shown in Figure 3 (right). As we can see, the survey resolution is increased, while noise associated with the downward continuation procedure is removed, since it is mostly concentrated in a very high frequency range of the spectrum.

\section{Application of the total normalized gradient in depth estimations of burial objects at SWSA 6.}

Several profiles going across dominant strike direction were chosen inside SWSA 6 area for the next interpretation.

Magnetic data Results of applying TNG to the pole reduced magnetic data for the profile 1 are shown in Figure 4 (left). The downward continued magnetic data are shown in Figure 4 (right). The depth estimates for downward continued data are moved up a distance approximately equal to the flight altitude, and the resolution of the image in the z-direction is enhanced. For example, a deep TNG anomaly on the left side of the cross-section corresponds with the hypothesis of a karst hole, filled with the highly magnetic liquids. Depth estimates generally correspond with depths of well known buried objects at SWSA 6 quite well.

AEM data The Oak Ridge AEM survey recorded data at frequencies: $850,4000,32000 \mathrm{kHz}$. Both coplanar and coaxial loop configurations were used. Only the horizontal loop configuration was used for the TNG transformation. Among these frequencies $4000 \mathrm{~Hz}$ was chosen as the most sensitive to subsurface targets, while not distorted by such noise sources as power lines The same profiles across SWASA6 as in a case of the airborne magnetic survey were processed using TNG gradients. Figure 5 shows results of this processing applied to the Profile 1. AEM data are useful complements to the magnetic data and in some cases depict targets that don't have magnetic anomalies associated with them. 


\section{Conclusions}

The airborne geophysical survey carried of the Oak Ridge Reservation has shown that AEM can be used in evaluating details of waste areas. However, to detect relatively small objects, a few drums, for example, the flight altitude must be kept in a range of $10-15$ meters. Due to the natural obstacles present in the Oak Ridge area, this requirement is impossible to achieve in many cases, and a sensor height of $30 \mathrm{~m}$ or more must be attained. In these conditions the data processing involving downward continuation allows to improve the survey resolution, and might be considered as a pseudo sensor height reduction. The total normalized gradient provides an additional information about the depth of buried objects. The most striking difference between the TNG method and most other depth estimation methods is that the total normalized gradient technique involves only a computational straightforward process of downward continuation, as opposed to the solution of a system of equations for source parameters. The method has essentially no adjustable parameters and can be used in a semi-automatic mode.

\section{References}

Beard, L.P., Nyquist, J.E., and Doll, W.E., 1995, High resolution airborne geophysics at hazardous waste disposal sites: SAGEEP'95 proceedings, 647-656.

Berezkin, V.M., 1973, Method of total normalized gradient in geophysical exploration: Nedra, Moscow $189 \mathrm{pp}$.

Doll, W.E., Helm, J.M., and Beard, L.P., 1995 Airborne detection of magnetic anomalies associated with soils on the Oak Ridge reservation, Tennessee: SAGEEP'95 proceedings, 619-626.

Nabighian, M., 1972, The analytic signal of two-dimensional magnetic bodies with polygonal crosssection: its properties and use for automated anomaly interpretations: Geophysics, 37, 507-517.

Nabighian, M., 1974, Additional comments on the analytic signal of two-dimensional magnetic bodies with polygonal cross-section: Geophysics, 39 85-92.

Roest W.R., Vehoef J., and Piklington M., 1992 Magnetic interpretation using the 3-D analytic signal: Geophysics, 57, 116-125.

Xiong, Z., 1992, Electromagnetic modelling of three-dimensional structures by the method of system iterations using integral equations, Geophysics, 57, 1556-1561. 

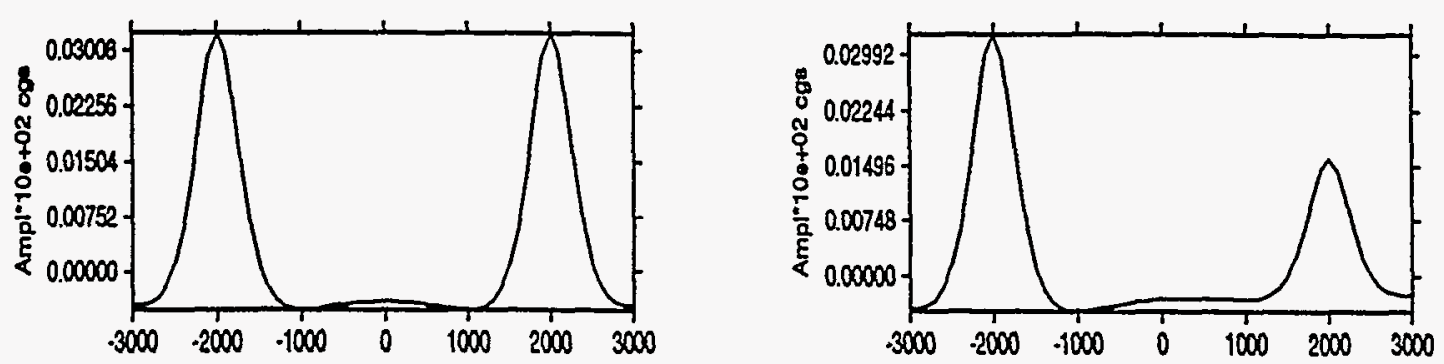

Total Normalized Gradient Field
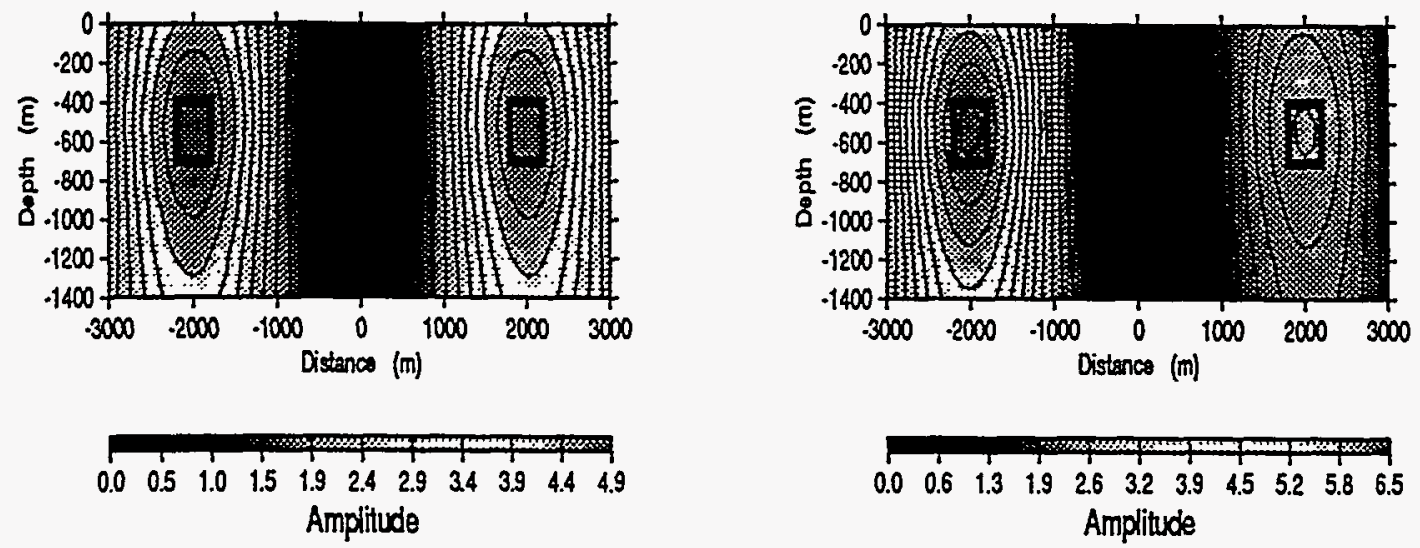

Figure 1: Maximum of the total normalized gradient for relatively small isometric objects coincide with the mass's center: two magnetic bodies with the same magnetic susceptibility (left), and two magnetic bodies withe magnetic susceptibility of the second body reduces from $250 \mathrm{cgs}$ to $125 \mathrm{cgs}$ (right). 
20 interpretation using ING

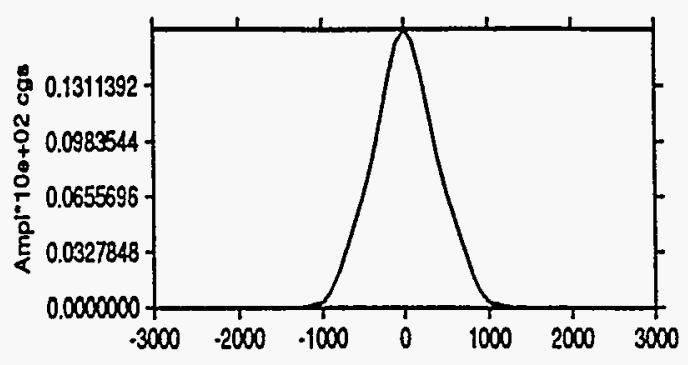

Total Normalized Gradient Field
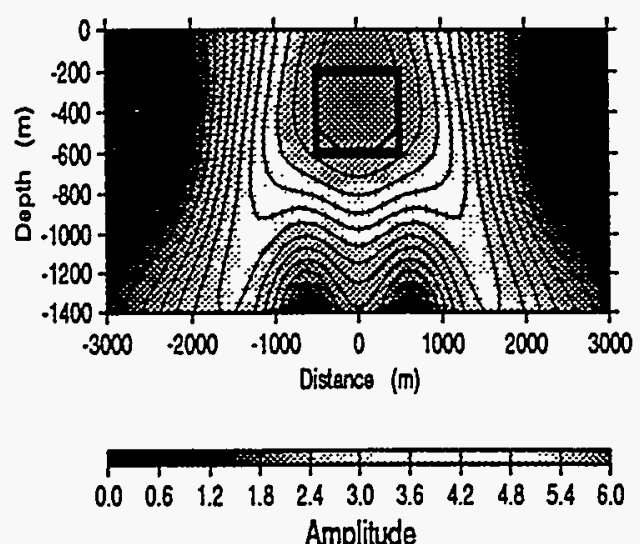

$2 D$ interpretation using $\mathbb{T N G}$

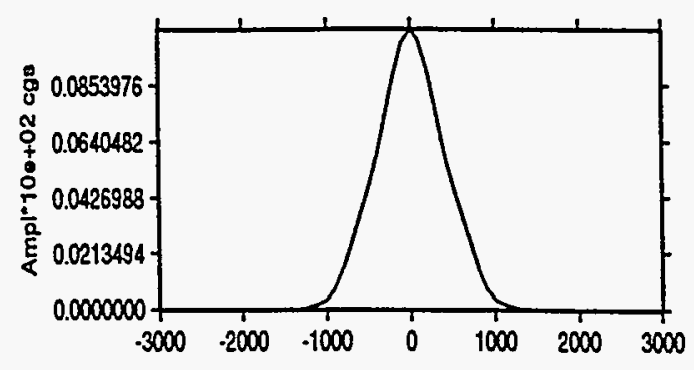

Total Normalized Gradient Field

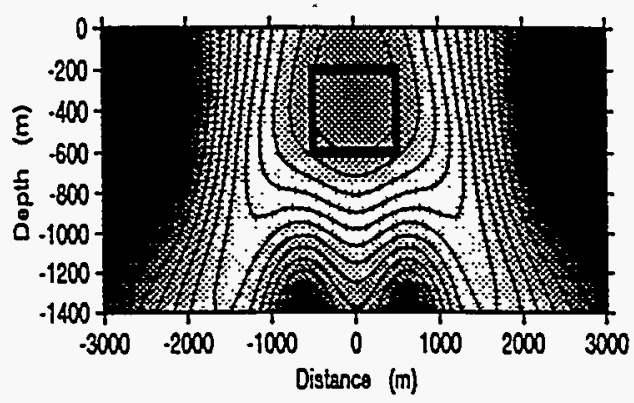

$\begin{array}{llllllllllllll}0.0 & 0.6 & 1.2 & 1.8 & 2.4 & 3.0 & 3.6 & 4.2 & 4.8 & 5.4 & 5.0\end{array}$ Amplitude

Figure 2: Results of using the total normalized gradient method on frequency-domain $(4 \mathrm{kHz})$ synthetic data computed at the earth surface (left) and at the flight altitude $50 \mathrm{~m}$ (right) 

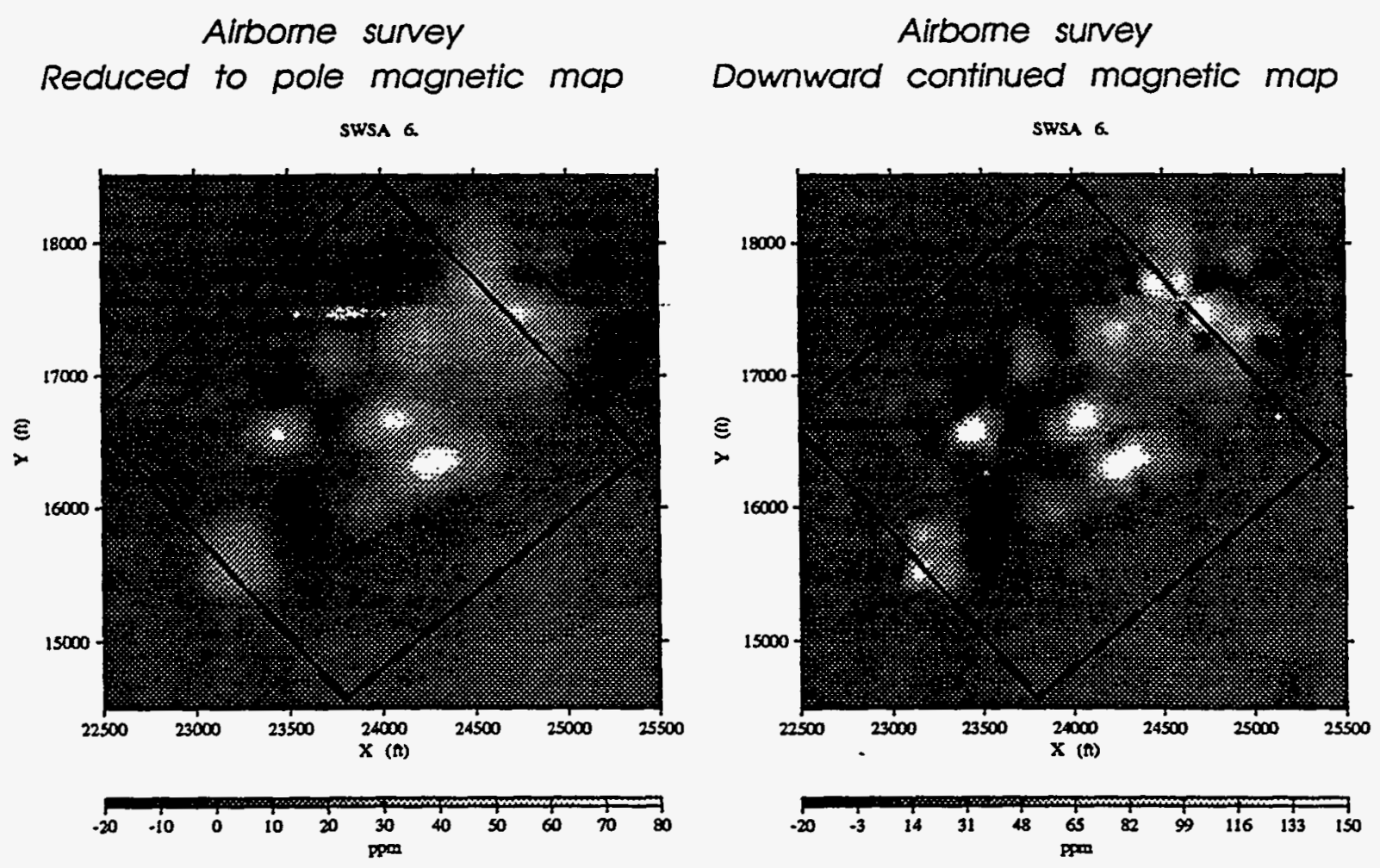

Figure 3: The reduced to pole magnetic map of the SWSA 6 area (left) and the smae map, filtered and downward continued at $75 \mathrm{ft}$ (right) 
Magnexic filed. SHSA 6. Profile 1.

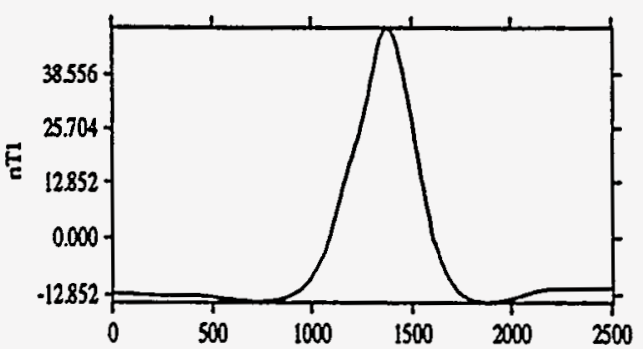

Total Normalized Gradieat Field

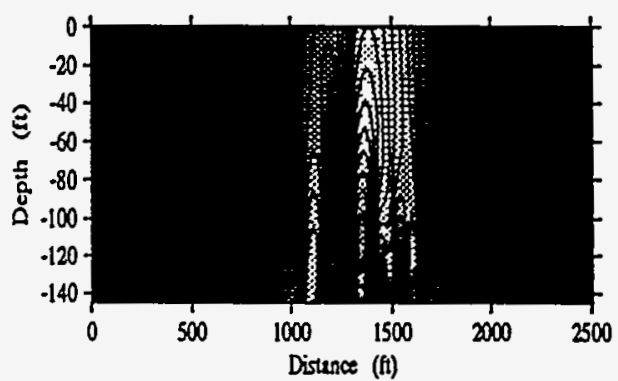

$\begin{array}{llllllllllll}0.0 & 0.7 & 15 & 2.2 & 3.0 & 3.7 & 45 & 5.2 & 6.0 & 6.7 & 75\end{array}$ Amplibude
Downterd continged magnetic filed. SPSA 6. Profile

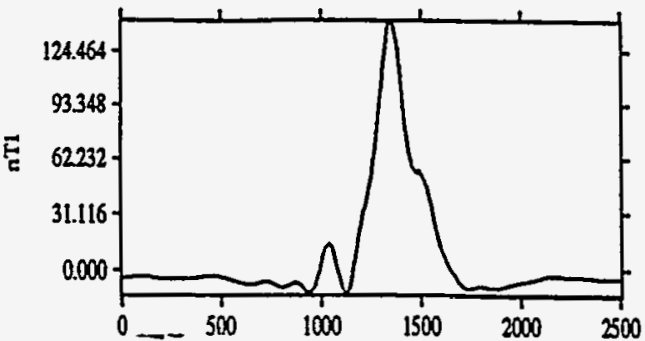

Total Namalized Gradient Fitd
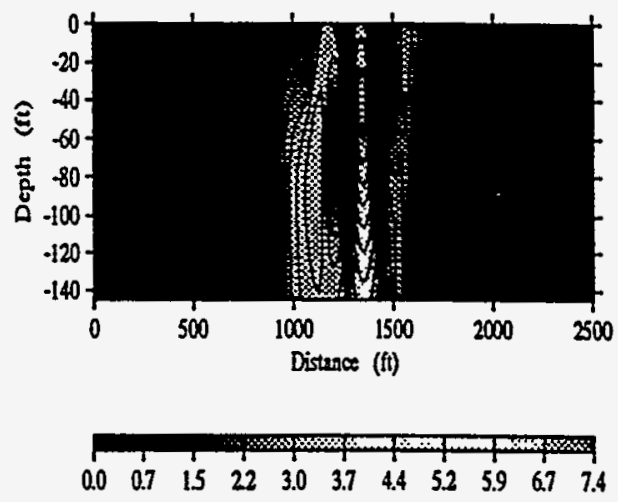

Amplitude

Figure 4: Results of using TNG to the reduced to pole magnetic data for the profile 1 (left), and Results of using TNG to the reduced to pole, filtered, and downward continued magnetic data for the same profie (right) 
Inphase part of EM field. $4189 \mathrm{~Hz}$. SWSA 6. Profile $1 .$.

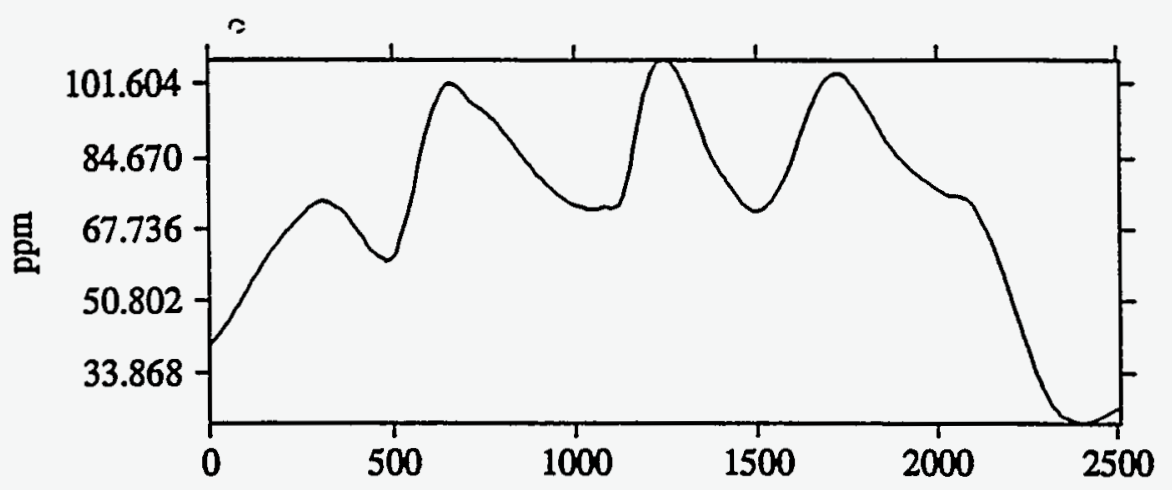

Total Normalized Gradient Field
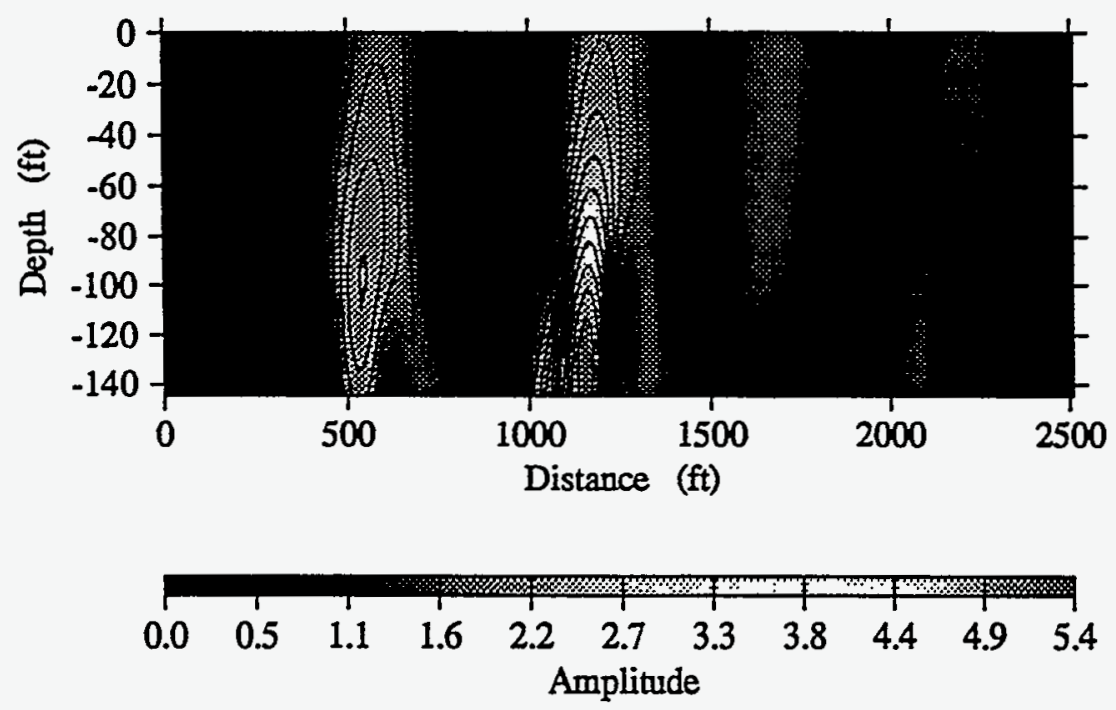

Figure 5: Results of using TNG to EM frequency $(4.2 \mathrm{kHz})$ inphase data over the profile 1 


\section{DISCLAIMER}

This report was prepared as an account of work sponsored by an agency of the United States Government. Neither the United States Government nor any agency thereof, nor any of their employees, makes any warranty, express or implied, or assumes any legal liability or responsibility for the accuracy, completeness, or usefulness of any information, apparatus, product, or process disclosed, or represents that its use would not infringe privately owned rights. Reference herein to any specific commercial product, process, or service by trade name, trademark, manufacturer, or otherwise does not necessarily constitute or imply its endorsement, recommendation, or favoring by the United States Government or any agency thereof. The views and opinions of authors expressed herein do not necessarily state or reflect those of the United States Government or any agency thereof. 